
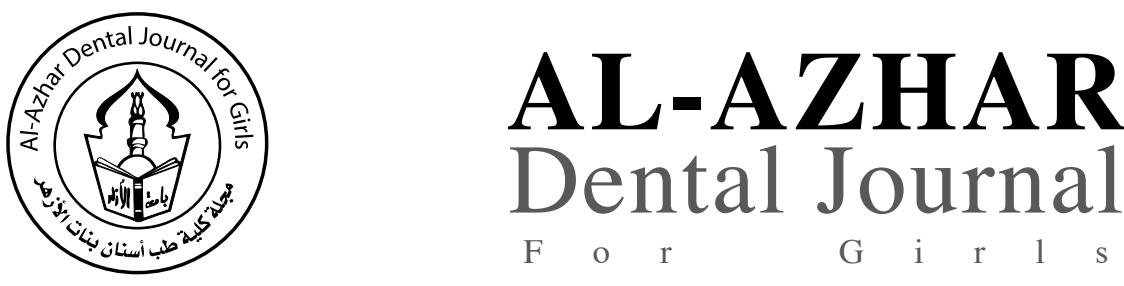

The Official Publication of The Faculty of Dental Medicine For Girls, Al-Azhar University Cairo, Egypt.

ADJ-for Grils, Vol. 3, No. 4, October (2016) — PP. 299:307

\title{
The Diagnosis of Neuromuscular in Orthodontics Effects of TENS on The Antero-Posterior Maxilla-Mandibular Relationship
}

\author{
Yasser Ali Ahmed Thabet ${ }^{(1)}$, Abumadyan Nasser Ahmed Aqrabe ${ }^{(1)}$, \\ Redwan Alasimy (2) and Mohamed Sobhy Mohamed ${ }^{(3)}$
}

Codex : 07/1610

dentaljournal.forgirls@yahoo.com

\begin{abstract}
Objective:This study was conducted in order to assess the changes in the occlusal position of the mandible after ULF (Ultra Low Frequency)- TENS relaxing procedure in subjects in pubertal growth phase with diagnosed Angle Class II division I and mandibular dentoalveolar retrusion. Materials and methods: This study was performed on 70 patients (48 females, 22 males) with an Angle Class II division I, aged between 10 and 15 years old (mean age 12.26, SD 1.32) characterized by mandible dentoalveolar retrusion and optimal vertical facial dimension, diagnosed by clinical and cephalometric evaluation. Diagnostic neuromuscular registrations were made for all subjects. The casts were mounted on articulator in habitual intercuspal position with a tooth-guided wax bite registration. Reference points were chosen at molar level. Subsequently the same casts were mounted in myocentric position and compared to the habitual intercuspal position, assessing the antero-posterior shift after TENS procedure. Statistics: Mean and standard deviation were calculated on the amount of shifting at the left molar reference point after TENS procedure. Analysis of variance was carried out in order to evaluate the influence of sex and age on the amount of molar shift. Results: Nine subjects showed, in the antero-posterior plane, a forward mandibular shift in neuromuscular myocentric position compared to habitual intercuspal position. Six subjects showed no differences between habitual and myocentric position in the antero-posterio plane. Four individuals showed a backward mandible shift after TENS indicating worsening of the II molar in the antero-posterior plane. Conclusion: This study suggests that TENS recorded occlusion in subjects with Class II division I with mandible dentoalveolar retrusion allows to visualize an unusual trend of growth The advancement of the mandible were not taken into account. These results could offer new diagnosis and prognosis methods for Class II malocclusions.
\end{abstract}

1. Associate Professor in Orthodontics Department, Faculty of Dentistry, Ibb University .

2. Associate Professor in Restorative Department, Faculty of Dentistry, Sana'a University.

3. Professor in Removable Prosthodontics Department, Faculty of Dentistry, Gazan University. 


\section{INTRODUCTION}

The growth potential of individuals with Class II malocclusions is of interest in orthodontic practice because these malocclusions represent a significant percentage of orthodontic cases ${ }^{(1)}$.In several cephalometric studios it has been observed that the relationship between maxilla and cranial base shows no significant differences in Class II division I compared to normal groups ${ }^{(2)}$. Furthermore other works suggested that the mandible is significantly backward positioned in Class II division I individuals compared to normal subjects ${ }^{(3)}$. Some investigated that in $96 \%$ of the subjects the total mandible length was similar in Class II subjects and in normal subject's age and sex matched ${ }^{(4)}$. They concluded that the difference in the bony bases is not the result of an abnormal development of any specific part but, rather, of an abnormal relationship between upper and lower jaws.

There is great individual variation in the mandibular linear growth ${ }^{(5)}$ and a high degree of variability is seen in Class II growth pattern of mesofacial brachifacial subjects. Frequently, the existing habitual occlusion is not an optimal relationship between the mandible and the cranium. Therefore, an orthodontic class diagnosis based on intercuspated casts or cephalometric radiographs could be misleading because of the incorrect and, frequently, "pathological" (6).

Several years ago, different authors emphasized the importance of rest position and its reproducibility as a diagnostic ${ }^{(7-9)}$. The idea of rest position was not helpful until the introduction of new modalities for relaxing muscles and recording the occlusal position based on relaxed muscles. With the development of the Myo-monitor, Ultra Low Frequency Transcutaneous Electrical Stimulation (ULF-TENS) of the motor branches of the $\mathrm{V}$ and VII cranial nerves the rest position became clinically useful ${ }^{(10-12)}$. The primary goal of TENS is to relax mandible and facial musculature in order to identify and establish the mandible rest position with relaxed muscles. Starting from this reference position, ULF-TENS allows recording of a muscle-driven occlusal position, called Myocentric position.

Despite this interesting point of view, literature shows a lack of evidence on the effect of ULFTENS in orthodontic patients suffering from Class II division I, and no comparisons have been made between classical growth prediction and maxillomandibular relationship driven by muscles and based on ULF-TENS

This study was conducted in order to assess the changes on the antero-posterior plane of the occlusal position of the mandible after ULF (Ultra Low Frequency)- TENS relaxing procedure in children with diagnosed Class II division I and mandibular dento-alveolar retrusion.

\section{MATERIALS AND METHODS}

This study was performed on 70 patients (48 females, 22 males) with Angle Class II division I, aged between 10 and 15 years, with a mean age of 12.26 years (SD 1.32). The subjects were selected from an initial group of 110 Class II division I patients with mandibular dentoalveolar retrusion, absence of deviation of mandibular and of anterior tooth midlines diagnosed by clinical examination, lateral and frontal teleradiographs.

The subjects were chosen according to the following criteria: retruded chin in the sagittal plane ( with respect to the line perpendicular to Frankfort plane through Nasion) at the clinical examination; alignment of upper and lower interincisve lines and II molar/canine Class symmetries at the intraoral examination; absence of pain, noise, and tenderness referred to TMJ at the functional examination. Sagittal teleradiographs confirmed Class II division I with mandibular dentoalveolar retrusion and optimal vertical facial dimension. Frontal teleradiographs, taken with the open mouth, confirmed the symmetry of maxillary and mandibular structures. In order to be considered for the study the subjects had to exhibit the following characteristics. 
1. Angle Class II division I with mandible dentoalveolar retrusion according to the APog line (overjet 5.5-10 $\mathrm{mm}$ ).

2. ANB angle $>4 \mathrm{~mm}$.

3. Optimal vertical dimension ( $\mathrm{SnGoGn} 26-38$ degree).

4. No history of orthodontic treatment.

5. No observable or $<1.5 \mathrm{~mm}$ deviation of anterior tooth midlines.

6. Absence of skeletal asymmetry.

7. Absence of anterior or posterior/lateral cross-bite.

8. Non- extraction treatment plane.

The patients were in the pubertal growth phase and had development potential. The subjects had completed an average of $90.81 \%$ of their growth potential. Lateral cephalometric radiographs were obtained at the beginning of the study ${ }^{(6)}$.

\section{Experimental protocol}

Diagnostic neuromuscular registrations were carried out in 45 children. Accurate dental casts were mad for each patient. For each cast, reference points marked: the line of inter-incisors maxillary side (fig. 1b), and the line perpendicular to the antero-posterior plane on molar right and left sides (fig. 1a, 1c). As the molar to molar position did not follow the same trend, because of the tridimensional changes of the occlusal relationship induced by TENS, the cases showing a difference between right and left molar reference points $>0.5 \mathrm{~mm}$ were excluded.

This simplified procedure allowed to consider only the individuals with symmetric effect of ULFTENS on muscular relaxed myocentric occlusion. In order to evaluate the antero-posterior modification, only the right molar ( fig. 1b and c) was considered as reference point, because in this work only the cases presenting symmetric mandible movement after TENS are considered. The Myo- monitor (transcutaneous electro-neural stimulation, j5 Myotronics-Noromed,inc., Tukwila, WA) was used to relax the mandible musculature. The resulting rest position was clinically measured and recorded. An acrylic registration wax made (myoprint).

Table (1) The intra-observer methodological error $s$ (i) is calculated from duplicate recordings. $S$ denotes the variance of the total sample of 70, with the values of the left and right sides combined.

\begin{tabular}{ccc}
\hline & S (i) & s(i)Ç\% 0f SÇ \\
\hline Molar relation & 0.6 & 3.1 \\
\hline
\end{tabular}

When the acrylic has hardened in its plastic phase it was removed from the mouth, undercuts were eliminated and it was placed between the casts, allowing for complete hardening. Several acrylics were realized to check the accuracy of the registration. The casts were mounted on Galetti ( Sevtis-Fisat s.r.l. plastic technology) articulator and the mounting was checked for accuracy with the myoprint which had been used for mounting. With the subjects' casts mounted at the myocentric position, the possible shift of the mandible reference points was calculated and analyzed by means of digital gauge comparing habitual to myocentric occlusion. All measurements were performed twice and the intra-examiner error was calculated by the formula $\mathrm{s}(\mathrm{i})=\sqrt{ } \Sigma \mathrm{d}^{2} / 2 \mathrm{n}$.

\section{Statistical analysis}

Mean and standard deviation were assessed on the amount of molar shift after TENS procedure.

A fast-setting acrylic material was placed over the occlusal surfaces of the lower teeth, while the patient stood in a relaxed mandible posture induced by ULF-TENS. When the acrylic material was placed on the lower teeth the amplitude of TENS was increased to passively lift the mandible from rest position to myocentric position, $1.5-2.0 \mathrm{~mm}$, cranial to the rest position. 


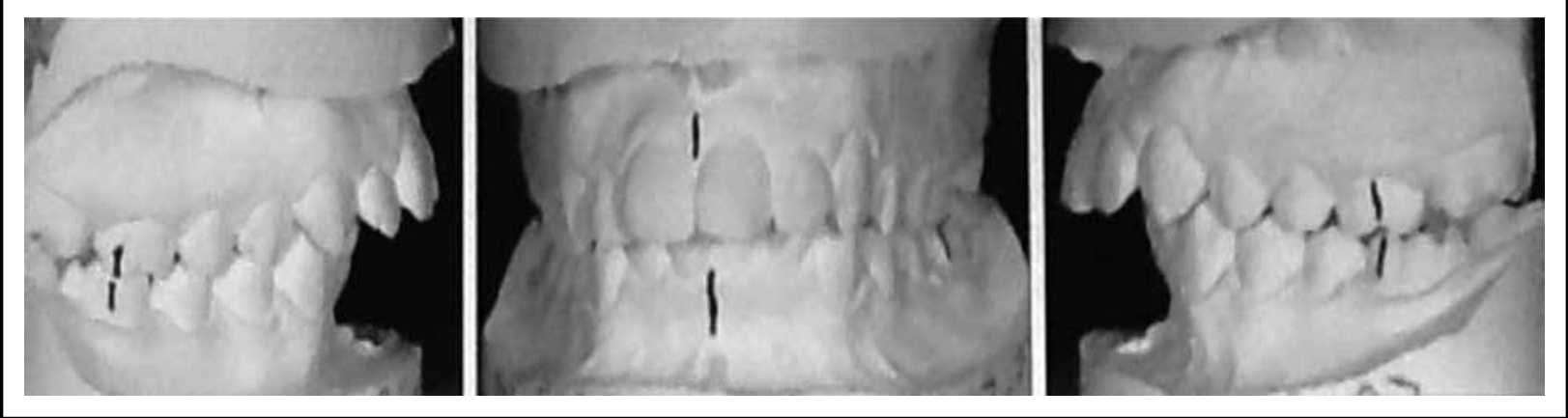

Fig. (1) Dental casts mounted in habitual ocelusion: a) right molar side; b) incisal side, c) left molar side
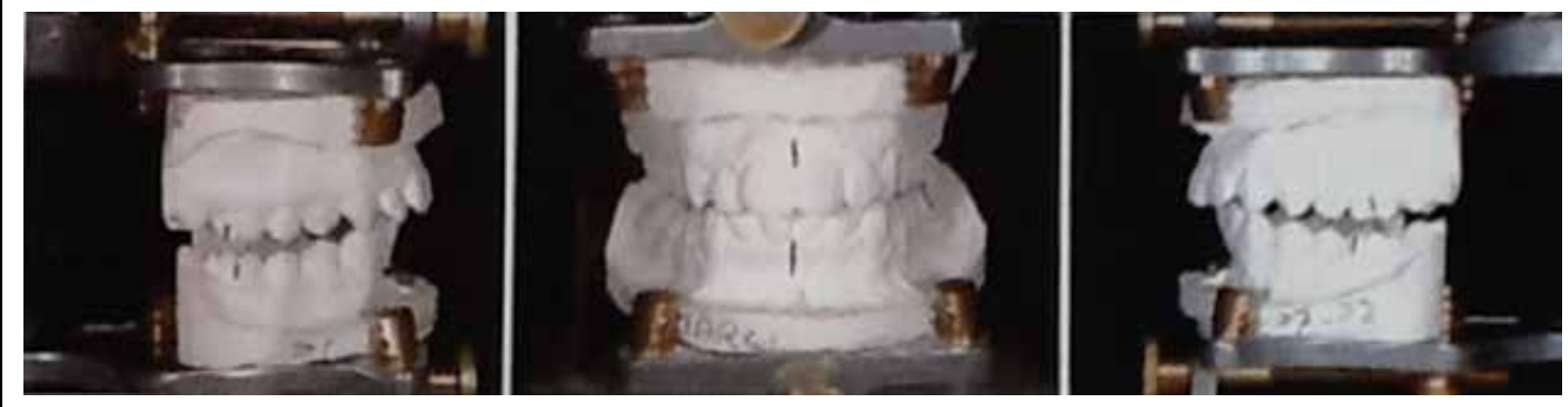

Fig. (2) Casts mounted in myocetric position: a) right molar side; b) incisal side, c) molar side
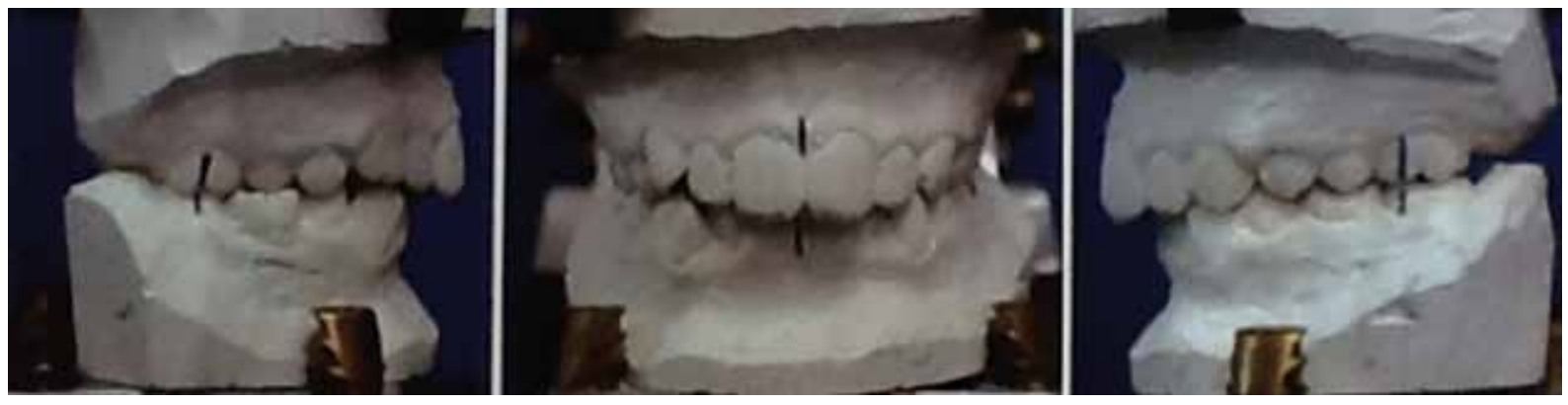

Fig. (3) Dental mounted in habitual occlusion: a) right molar side; b) incisal side, c) molar side
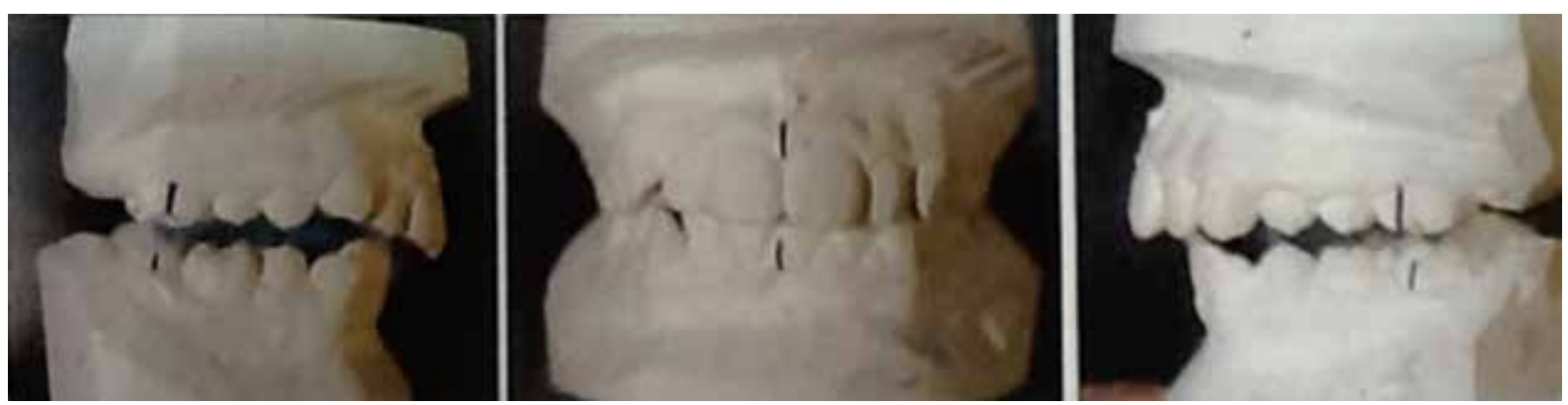

Fig. (4) Casts mounted in myocelric position: a) right molar side; b) incisal side, c) molar side 


\section{RESULTS}

The intra-examiner methodological error s (i) is presented in table 1 and mean repeatability and reliability of measurements.

Table 2 describes the composition of the group. Showing sex and age of subjects. Seventy individuals, 22 males and 48 females (mean age 12.26 , SD 1.32) were included in the study.

Table 3 shows the amount of the first right lower molar sagittal shift after TENS compared to its habitual occlusion position. The amount of shift in millimeters is preceded the sign + if the shift was medial, and considering that the sample comprised only individuals with symmetric TENS, shift in this case TENS induced a forward movement of jaw; when the sign-precedes the amount of shift, TENS induced a backward movement of jaws. When TENS did not change the antero-posterior relationship between upper and lower arches, the amount of shift was 0 .

Table (2) Age and gender of subjects

\begin{tabular}{|l|l|l|}
\hline 70 Subjects & Age & Sex \\
\hline SA & 11 & Female \\
\hline NS & 10 & Female \\
\hline IA & 15 & Male \\
\hline AA & 14 & Male \\
\hline SHS & 12 & Female \\
\hline LA & 11 & Female \\
\hline KHA & 12 & Female \\
\hline MG & 13 & Male \\
\hline SHA & 12 & Female \\
\hline RG & 11 & Female \\
\hline ZA & 13 & Male \\
\hline SHAL & 12 & Female \\
\hline AM & 11 & Female \\
\hline HA & 14 & Male \\
\hline BS & 11 & Female \\
\hline RM & 12 & Female \\
\hline SH & 13 & Female \\
\hline HH & 12 & Female \\
\hline EA & 14 & Male \\
\hline MA & 15 & Female \\
\hline SB & 12 & Female \\
\hline FD & 11 & Female \\
\hline FE & 11 & Male \\
\hline & & \\
\hline
\end{tabular}

\begin{tabular}{|c|c|c|}
\hline 70 Subjects & Age & Sex \\
\hline MS & 14 & Female \\
\hline MN & 15 & Female \\
\hline $\mathrm{GH}$ & 11 & Male \\
\hline HD & 12 & Female \\
\hline NA & 13 & Female \\
\hline OS & 12 & Male \\
\hline ZM & 14 & Female \\
\hline SA & 11 & Female \\
\hline TS & 10 & Male \\
\hline MM & 11 & Female \\
\hline $\mathrm{HS}$ & 10 & Female \\
\hline MD & 12 & Male \\
\hline FS & 12 & Female \\
\hline GM & 11 & Female \\
\hline DA & 13 & Female \\
\hline $\mathrm{AD}$ & 15 & Female \\
\hline YS & 15 & Male \\
\hline LY & 14 & Female \\
\hline GS & 11 & Female \\
\hline NA & 13 & Female \\
\hline SHA & 10 & Male \\
\hline DS & 12 & Female \\
\hline $\mathrm{RF}$ & 11 & Male \\
\hline RA & 13 & Female \\
\hline NS & 15 & Female \\
\hline GF & 10 & Male \\
\hline MG & 11 & Female \\
\hline KA & 13 & Male \\
\hline DB & 12 & Female \\
\hline DHA & 10 & Female \\
\hline MM & 15 & Female \\
\hline SY & 11 & Male \\
\hline $\mathrm{NZ}$ & 15 & Female \\
\hline $\mathrm{NH}$ & 14 & Female \\
\hline $\mathrm{RN}$ & 12 & Female \\
\hline KHF & 13 & Male \\
\hline HA & 10 & Female \\
\hline TA & 15 & Female \\
\hline TS & 14 & Male \\
\hline AN & 15 & Female \\
\hline $\mathrm{HN}$ & 13 & Female \\
\hline MR & 12 & Female \\
\hline AR & 13 & Male \\
\hline FY & 10 & Male \\
\hline IN & 12 & Female \\
\hline ZM & 15 & Male \\
\hline $\mathrm{ZN}$ & 11 & Female \\
\hline
\end{tabular}


Table (3) The amount of molar shift for each subject.

\begin{tabular}{|c|c|}
\hline 70 Subjects & Shift amount \\
\hline SA & +2.5 \\
\hline NS & 0 \\
\hline IA & 0 \\
\hline AA & +1.5 \\
\hline SHS & -1 \\
\hline LA & 0 \\
\hline KHA & 0 \\
\hline MG & +3 \\
\hline SHA & +1.5 \\
\hline $\mathrm{RG}$ & -2 \\
\hline $\mathrm{ZA}$ & +3 \\
\hline SHAL & 0 \\
\hline AM & 0 \\
\hline HA & +3 \\
\hline BS & +2 \\
\hline RM & +2.5 \\
\hline SH & +2 \\
\hline $\mathrm{HH}$ & -1.5 \\
\hline EA & -3 \\
\hline MA & 0 \\
\hline SB & +1.5 \\
\hline FD & 0 \\
\hline FE & -3 \\
\hline MS & 0 \\
\hline MN & +1.5 \\
\hline $\mathrm{GH}$ & +3 \\
\hline HD & 0 \\
\hline NA & 0 \\
\hline $\mathrm{OS}$ & +2 \\
\hline $\mathrm{ZM}$ & +2.5 \\
\hline SA & +3 \\
\hline TS & -1.5 \\
\hline MM & -2 \\
\hline HS & -3 \\
\hline MD & +3 \\
\hline FS & +1.5 \\
\hline GM & +2.5 \\
\hline DA & 0 \\
\hline $\mathrm{AD}$ & 0 \\
\hline YS & -2.5 \\
\hline LY & +2.5 \\
\hline GS & +1 \\
\hline NA & -2 \\
\hline SHA & +1.5 \\
\hline DS & +1 \\
\hline RF & +3 \\
\hline $\mathrm{RA}$ & +2 \\
\hline NS & +1 \\
\hline $\mathrm{GF}$ & -1 \\
\hline MG & 0 \\
\hline KA & 0 \\
\hline DB & +3 \\
\hline DHA & +2 \\
\hline MM & 0 \\
\hline SY & +2 \\
\hline $\mathrm{NZ}$ & +1.5 \\
\hline $\mathrm{NH}$ & +3 \\
\hline RN & 0 \\
\hline KHF & -1 \\
\hline HA & $\frac{1}{-2}$ \\
\hline TA & +2 \\
\hline TS & 0 \\
\hline $\mathrm{AN}$ & +1 \\
\hline $\mathrm{HN}$ & +1 \\
\hline MR & +3 \\
\hline $\mathrm{AR}$ & +1 \\
\hline FY & -1 \\
\hline IN & 0 \\
\hline $\mathrm{ZM}$ & -1.5 \\
\hline $\mathrm{ZN}$ & -3 \\
\hline
\end{tabular}

Table (4) Mean (Standard Deviation in parentheses) and direction of molar shift for all subjects

\begin{tabular}{|c|c|c|c|c|}
\hline Shift & Subject & $\mathrm{N}^{\circ}$ & $\%$ & Mean shift \\
\hline Mesial & 30 & 47.4 & 2.375 & $0.64 \mathrm{~mm}$ \\
\hline Distal & 15 & 21.1 & 1.875 & $0.85 \mathrm{~mm}$ \\
\hline Unchanged & 25 & 31.6 & \multicolumn{2}{|l}{} \\
\hline
\end{tabular}

Table (4) shows the sample summary statistics of the shift after TENS. After TENS procedure the right lower molar line shows an anterior average shift compared to the habitual occlusion of $2.375 \mathrm{~mm}$ (SD 0.64) in 30 subjects; a posterior average shift of $1.875 \mathrm{~mm}$ (SD 0.85) in 15 subjects; 25 individuals did not change the sagittal relationship between the arches after TENS procedure.

The right molar-line after TENS shifted mesially in 30 individuals, in 15 subjects it shifted distally and in 25 it did not change position.

In other word, after TENS 30 individuals reduced the Class II molar/canine relationship; 25 of them achieved a Class I molar/canine relationship, 15 subjects worsened the Class II relationship and 25 did not change class.

\section{DISCUSSION}

In our sample the TENS procedure, based on neuromuscular relaxation of the muscle supplied by the V and IIV cranial nerves, showed that 25 individuals changed the habitual craniomandibular relationship advancing the right mandibular molar line. Five individuals did not change position and 15 subjects showed a more distal relationship between the arches compared to the habitual relationship. In these last 40 cases neuromuscular procedure disagreed with classic orthodontic diagnosis. Indeed, these results did not confirm those on growth prediction ${ }^{(13)}$, in which the normal mandibular angle ( FMA $+/-4^{\circ}$ ) is found to be associated with balanced forward and downward development of the mandible ${ }^{(14)}$. 
Neuromuscular TENS procedure suggested that maxillo-mandibular relationship could change in unpredictable way, confirming that static parameters, such as molar Class, are not a sufficient criterion to drive a orthodontic diagnosis and therapy based on functional demands. Our data disagree with the objection that ULF-TENS procedure often determines a downward and forward displacement of the mandible ${ }^{(15-17)}$, and agree with the previous work by Monaco et al., confirming that the posterior areas of the mandibular arch could move in a very unpredictable way, resulting in individual and unpredictable diagnosis and prognosis of mandibular lateral deviation ${ }^{(18)}$.

In this study 40 subjects did not show any downward and forward displacement of mandible after TENS. These subjects represent the 52\% of the entire sample. Moreover, after TENS procedure the myoprint position could disagree with the classical orthodontic prediction of growth. Frequently, the mandible moves in an unpredictable way. Considering the results of the study, the standard mesial movement $(6 \mathrm{~mm}$ forward and 2 $\mathrm{mm}$ downward from habitual occlusion) suggested to fabricate the functional activators could be misleading. On the contrary, our data suggest that neuromuscular technique could offer the specific mandible shift on the sagittal plane needed for each subject characterized by Class II division I with mandibular dentoalveolar retrusion and optimal vertical facial dimension.

Our work demonstrates that it's impossible to take for granted that after TENS there is a high reduction of maxillo-mandibular discrepancy. Some individuals showed improvement in mandible retrusion, some worsened and others did not change the molar relationship.This result suggests that, from a neuromuscular point of view, Class II division I could be an heterogeneous diagnostic group and it proposes that a different approach based on muscle balance is needed in order to plan a functional orthodontic treatment.
The habitual or clinical relationship between dental arches is based on voluntary action by the patient, with or without mandibular guidance, or on manipulation exerted by the orthodontist. According to the neuromuscular data presented in this work, such a relationship may, but sometimes may not, show the truly relaxed relationship between mandible and maxilla ${ }^{(19)}$.

The observation made in our work could suggest a different treatment in subjects responding with forward shift after TENS comparing to individuals responding with backward shift or without any shift. In the first case (forward shift) a functional appliance which displaces the mandible forward according to the myoprint recording could be advisable in order to obtain a muscle balanced treatment.

In the other cases ( backward shift and no shift ) forward displacement of the mandible does not agree with muscle balance and could be unfavorable for orthodontic treatment. Such a forward displacement could be absolutely harmful for muscle balance if after TENS procedure the mandible shifts backward. In this case standard orthodontic device, prepared in order to advance the mandible from habitual occlusion, clashes with muscle forces. According to the data presented in this study this kind of approach could not be classified as "functional".

A basic reason is opposed to correct in a forward forced standard position people who respond to TENS with backward shift. In these subjects one of the common causes of mandible retrusion may lie in altered neuromuscular information coming from a peripheral somatic dysfunction, for example related to delivery trauma ${ }^{(20-24)}$.

Clinicians have repeatedly emphasized the need to fulfill requirements for normal muscle and temporomandibular joint function in addition to aesthetic alignment of the dental arches. The efficacy of low frequency TENS to achieve resting length of muscles supplied by the V and VII cranial nerves provides an ideal tool for the orthodontist to diagnose normal, rather than pathologic, mandible 
rest position and the related occlusal position ${ }^{(25)}$. Our data agree with those of ${ }^{(19)}$ who considers, after neuromuscular analysis, only certain functional discrepancies which became apparent. This may be of clinical significance and it stresses the importance of early neuromuscular diagnosis of these malocclusions, in order to be able to plan a correct and etiologic therapy of this complex malocclusion.

\section{CONCLUSION}

Recorded occlusion could be considered successful in both transverse and vertical dimension, and seems to be stable in the long term.

The aim of this work is to present the clinical approach in treating a case of class II division I malocclusion with maxillary constriction, the patient showed mouth breathing and finger sucking habits, (the diagnosis of the lateral deviation).

This study suggests that TENS recorded occlusion in subjects with Class II division I mandible dentoalveolar retrusion allows to visualize an unusual trend of mandible position.

The routine use of TENS could improve the diagnosis, the therapy and the prognosis of this complex orthodontic category, providing the clinician with a tool to study the individual treatment of the mandible.

Aim of these works (diagnosis of the lateraldeviations and diagnosis of the Angle Class II divisionI) is to provide a starting point to better understand the diagnostic role of TENS in pediatric orthodontics and dentofacial orthopedics. Surely several steps had to be done clarify the clinical protocol that could help clinicians in the resolution of such orthodontic and dentofacial orthopedic problems. Consequently, aim of a next work could be to analyse the growth prediction pattern before and after TENS procedure.

\section{REFERENCES}

1. Bishara SE. Mandibular change $s$ in persons with untreated and treated Class II division I malocclusion. Am J Orthod Dentofacial Orthop 1998; 113(6):661-73.

2. Rothstein T, Yoon-Tarlie C. Dental and facial skeletal characteristics and growth of males and females with Class II division I malocclusion between the ages of 10 and 14(revisted)-part 1: characteristics of size, form, and position. Am J Orthod Dentofacial Orthop 2000; 117(3):320-32.

3. Keski-Nisula K, Keski-Nisula L, Makela P, Maki-Torkko T, Varrela J. Dentofacial features of children with distal occlusions, large overjets, and deep bites in the early mixed dentition. Am J Orthod Dentofacial Orthop 2006;130(3):292-99.

4. Maj G, Alleva F. Lucchese FP. Changes in length and width of the mandibular arch from the mixed dentition completion of the permanent dentition. Eur J Orthod 1979; 1(4):259-63.

5. Gomes AS, Lima EM. Mandibular growth during adolescence. Angle Orthod 2006; 76(5):786-90.

6. Trahar M, Sheffield R, Kawamoto H, Lee HF, Ting K. Cephalometric evaluation of the craniofacial complex in patients treated with an intraoral distraction osteogenesis device: a preliminary report. Am J Orthod Dentofacial Orthop 2003 Dec; 124(6):639-50.

7. Thompson JR. Anatomical and Phsiological considerations for the positions of the mandible. Dent J Aust 1951 Apr; 23(4):161-66.

8. Perry HT, Harris SC. Role of the neuromuscular system in functional activity of the mandible. J Am Dent Assoc 1954 Jun; 48(6):665-73.

9. Mohamed SE, Christensen LV. Mandibular reference positions. J Oral Rehabil1985 Jul; 12(4):355-67.

10. Jankelson B. functional position of occlusion. J Prosthet Dent. 1973 Oct; 30(4):559-60.

11. Jankelson B. Neural conduction of the myo-monitor stimulus: A quantitative analysis. J Prosthet Dent. 1975; 34: 245-53.

12. Jankelson B., Radke JC. The myo-monitor: its use and abuse. Quintessence Int Dent Dig. 1978 Feb; 9(2):47-52.

13. Ruf S, Pancherz H. The mechanism of Class II correction during Herbst therapy in relation to the vertical jaw base relationship: a cephalometric roentgenographic study. Angle orthod 1997;67(4):271-76. 
14. Turchetta BS, Fishman LS, Subtelny JD. Facial growth prediction: a comparison of methodologies. Am J Orthod Dentofacial Orthop 2007; 132(4):439-49.

15. Azarbal M. comparison of Myo-monitor centric position to centric relation and centric occlusion. J Prosthet Dent 1977; 38(3):331-37.

16. Jimenez ID. Electromyography of masticatory muscles in three jaw registration positions. Am J orthod Dentofacial Orthop 1989;95(4):282-88.

17. Hickman DM, Cramer R. The effect of different condylar positions on masticatory muscles electromyographic activity in humans. Oral surg Oral Med Oral Pathol Oral Radiol Endod 1998;86(1):2-3.

18. Monaco A, Cattaneo R, Marci MC, Marzo G, Gatto R. Giannoni M. Neuromuscular diagnosis in orthodontics: effects of TENS on maxillo-mandibular relationship. Eur J Paediatr Dent 2007 Sept; 8(3):143-48.

19. Dinham G.A. Myocentric a clinical appraisal. Angle
Orthod 1983vol 54n $3:$ 211-17.

20. 20- Dunn PM. Congenital postural deformities. Br Med Bull. 1976 Jan; 32(1):71-6.

21. Pirttiniemi P, Lahtela P, Huggare J, Serlo W, head posture and dentofacial asymmetries in surgically treated muscular torticolis patients.Acta Odontol Scand 1989;47:193-97.

22. Pirttiniemi P, Gron M, Alvesalo L, Heikkinen T, Osborne R. Relationship of difficult forceps delivery to dental arches and occlusion. Pediatr Dent 1994 Jul-Aug; 16(4):289-93.

23. Harila-Kaera V, Gron M, Heikkinen T, Alvesalo L. Sagittal occlusal relationship and asymmetry in prematurely born children. Eur J Orthod 2002 Dec; 24(6):615-25.

24. Carttaneo R, Monaco A, Serafini V, Giannoni M. Birth Delivery Trauma and Malocclusion. J Clin Pediatr Dent 2005;12:123-30.

25. Jankelson Bernard: Neuromuscular aspects of occlusion Dental Clinis of North America 1979; 23, 2:1. 